divided. Suppose the section of Fresnel's wave surface by a coördinate plane to consist of a circle and an ellipse and let ( $A$ be the center of a wave disturbance in the surface of a crystal. Draw any radius vector $O B C$, cutting the circle at $B$ and the ellipse at $C$; the intersection of tangents at $B$ and $C$ will be $S$, a point on the tangent plane at the point of incidence. Then $S B$ and $S C$ will be the wave fronts of an ordinary and an extraordinary ray. Since these two lines are tangents at the points of intersection of a single radius vector $O B C$, the latter is the path of both an ordinary and an extraordinary ray. Therefore from the indices of refraction we may compute an incident ray which will not be divided but will move along $O B C$, and since there are an infinite number of points $S$ there are an infinite number of directions for which the ray is not divided. The locus of the point $S$ is a curve of the eighth degree and may be expressed in a simple and symmetrical form. By a simple transformation of the equation uniaxial crystals are included in this treatment.

Thomas F. Holgate, Acting Secretary.

Evanston, Illinors.

\title{
REPORT ON THE RECENT PROGRESS IN THE THEORY OF LINEAR GROUPS.
}

BY PROFESSOR L. E. DICKSON.

(Read before Section A of the American Association for the Advancement of Science, Columbus, Ohio, August 22, 1899.)

THIs report is intended to supplement in certain directions. the report* of Dr. Miller on group theory read at the last summer meeting of the American Association. In order to allow space for important details, I will consider neither continuous nor discontinuous linear groups of infinite order, but confine my report to finite linear groups. Of these I shall consider first the finite collineation groups and afterwards the linear congruence groups and the more general linear groups in Galois fields.

Part I.-Finite groups of linear substitutions.

\$1. General Theorems on Finite Linear Groups.

The following theorem was stated by Jordan in 1878 and proven in various ways by Lipschitz, Kronecker, Rost,

* Bulletin, Feb., 1899, p. 227. 
Maschke, Moore, * and recently by Burnside $\dagger$ in a very elementary manner :

A linear homogeneous substitution of finite period $p$;

$$
x_{i}^{\prime}=\sum_{i=1}^{n} \alpha_{i j} x_{j}, \quad(j=1,2, \cdots, n)
$$

may be linearly transformed to the canonical form

$$
y_{i}^{\prime}=\varepsilon_{i} y_{i}, \quad(i=1,2, \ldots, n)
$$

where each $\varepsilon_{i}$ is a $p^{\text {th }}$ root of unity.

Concerning the nature of the irrationalities which may enter into the coefficients of the substitutions of a finite linear group, we have the theorem due to Professor Maschke:-

If a linear substitution group of finite order contain at least one substitution, the roots of whose characteristic equation are all distinct, the group can be so transformed that all the coefficients of the substitutions of the transformed group are cyclotomic, $i$. e., are rationally expressible by roots of unity.

The existence of a universal invariant for finite linear groups is expressed by the theorem :-

A finite group of $n$-ary linear homogeneous substitutions leaves absolutely invariant a positive Hermitian form. By suitable linear transformation of the group this invariant becomes

$$
\sum_{i=1}^{n} x_{i} \bar{x}_{i}
$$

This theorem was stated by Loewy $\S$ and proven by Moore.|| The first part of the theorem was established earlier by Picard $\Phi$ for the cases $n=2,3$. The complete theorem was proven by Valentiner $* *$ for $n=2$ and 3 .

H. Minkowski $\dagger$ h has shown that every finite group $G$ of $n$-ary linear homogeneous substitutions with integral coefficients is isomorphic with the group of substitutions re-

* Math. Annalen, vol. 50, pp. 213-219. Full references are given in the paper by Professor Moore.

$\dagger$ Messenger of Math., no. 331 (November, 1898).

$\ddagger$ Math. Annalen, vol. 50, p. 492 .

8. Comptes rendus, vol. 123 (1896), pp. 168-171.

II Math. Annalen, vol. 50, pp. 213-219.

T Bull. Soc. Math de France, vol. 15 (1887), pp. 152-156.

** "De endelige Transformations-Gruppers Theot $i$," avec un resumé en français, Danish Academy, 1889.

t† Crelle, vol. 100 (1887), pp. 449-458. 
sulting upon reducing its coefficients modulo 2 , and derives the corollary that the order of $G$ is a divisor of $2^{n} N$, where $N \equiv(2-1)\left(2^{n}-2\right) \cdots\left(2^{n}-2^{n-1}\right)$ is the order of the group of all $n$-ary substitutions taken modulo 2 .

The generators of the group of $n$-ary linear substitutions of determinant unity and with integral coefficients were given by Kronecker* as follows :

$$
\begin{gathered}
x_{1}=-x_{k}^{\prime}, \quad x_{k}=x_{1}^{\prime}, \quad x_{i}=x_{i}^{\prime} \quad(i \gtrless k, i>1), \\
x_{1}=x_{1}^{\prime}+x_{2}^{\prime}, \quad x_{i}=x_{i}^{\prime} \quad(i=2,3, \cdots, n) .
\end{gathered}
$$

In place of these $n$ generators, Krazer $\dagger$ uses three generators. But Kronecker bad already shown that two generators would suffice, while the simple form of the generators would thereby be lost. For the case $n=3$, W. Burnside \$ proves that the generator (2) together with the substitution permuting $x_{1}, x_{2}, x_{3}$ in a cycle suffices to generate the group. In Kronecker's paper the generators of the abelian linear groups are also given.

\section{§2. Special Groups of .Collineations.}

The problem of the determination of all finite groups of binary linear substitutions has been solved geometrically by Klein $\S$ and algebraically by Gordan.\| Essentially the groups thus found are those connected with the regular solids. The finite ternary groups were considered at great length by Jordan, 9 who, however, missed the group of 168 collineations in the plane, which was derived by Klein ** by the consideration of the transformation of the seventh order of elliptic functions. He also missed the simple group of 360 collineations, set up by Valentiner t十 and proven simply isomorphic to the alternating group on six letters by Wiman. \$t Certain configurations connected with this $G_{360}$ had been given earlier by Gerbaldi. $\S$

* Monatsber. der Berl. Akad. (1866), p. 597.

† Annali di Matematica, 2d series, vol. 12, pp. 283-300.

$\ddagger$ Messenger of Math., 2d series, vol. 24 (1894), pp. 109-112.

\% Math. Annalen, vol. 9 (1876), pp. 183-188.

II Math. Annalen, vol. 12 (1877), pp. 23-46.

I Crelle, vol. 84 (1878), pp. 89-215; Atti della Reale Accademia di Napoli, vol. $8(1879)$.

** Math. Annalen, vol. 14, pp. 428-471 (Jordan's omission is indicated in the foot-note to p. 438); Math. Annalen, vol. 15, pp. 251-282.

$\dagger \dagger$ " De endelige Transformations-Gruppers Theori," avec un résumé en français, 1889, Danish Acaderny.

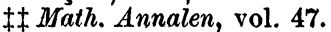

8. "Sul gruppo semplice di 360 collineazioni piane," Math. Annalen, vol. 50 , p. 472 . 
In view of the evident independence of the work of Jordan and Valentiner and their difference of method, we may feel certain of the completeness of the determination of the finite ternary linear groups. Of these groups the ones which leave a straight line invariant as well as those permuting three points cyclically are regarded as trivial. Besides these there exist only the groups of orders 216,168 , 360 , together with their subgroups of orders 72,36 , and 60 . We will briefly characterize the former set of three groups.

The Hesse group of 216 collineations is defined by a

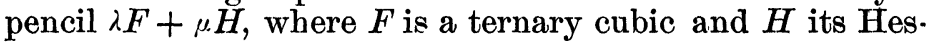
sian covariant. The $G_{216}$ has subgroups of orders 36 and 72. It has been studied at length by Maschke.*

The simple group of 168 collineations leaves invariant

$$
x^{3} y+y^{3} z+z^{3} x=0 \text {. }
$$

As mentioned above, it was first met by Klein. It is studied in detail in Klein-Fricke's Modulfunctionen. The types of substitutions and of subgroups of the $G_{168}$ are given by Gordan $\dagger$ (Mathematische Annalen, vol. 25, p. 462).

The simple group of 360 collineations has as its simplest invariant form the function

$$
10 x^{3} y^{3}+9 z\left(x^{5}+y^{5}\right)-45 x^{2} y^{2} z^{2}-135 x y z^{4}+27 z^{6} \text {. }
$$

It has two systems, each of 15 conjugate octahedral sulgroups, and two systems each of six conjugate icosahedral subgroups. The even permutations of the icosahedral groups of one such system form the alternating group on six letters (Wiman). The $G_{360}$ has ten conjugate subgroups of order 36. It is not possible to set up a group of 360 ternary homogenous substitutions holoedrically isomorphic with the group of 360 collineations. $\$$ This result follows from the corresponding impossibility for the subgroup $G_{36}$, as shown by Jordan.

Quaternary linear homogenous groups of orders 120 and 720 have been given by Klein, $\S$ one of order $2 \cdot 16 \cdot 6$ ! was studied by Reichardt. 9 All ternary and quaternary linear groups simply isomorphic to the symmetric or alternating group of substitutions have been determined by Maschke.**

* Math. Annalen, vol. 33.

† "Ueber Gleichungen siebenten Grades mit einer Gruppe von 168 Substitutionen:' I. Math. Annalen, vol. 20, pp. 516-530 ; II. Math. Annalen, vol. 25 , pp. 459-521.

† Wiman, Math. Annalen, vol. 47, p. 538.

Ikosaeder, p. 198 ; Math. Annalen, vol. 4.

Tl Leipzig Ber. (1885), pp. 419-426.

** Math. Annalen, vol. 51. 
The problem emphasized by Klein of representing every group as a collineation group in the fewest possible number of variables has been solved for the symmetric and alternating groups by A. Wiman.* $\mathrm{He}$ shows that, for $n>7$, no collineation group exists in space of $<n-2$ dimensions, which is isomorphic with the symmetric or alternating group on $n$ letters. Except for the case $n=9$, there is only one group in $R_{n-2}$ isomorphic with the symmetric and one with the alternating group on $n$ letters.

A quaternary linear homogeneous group of order 51840 arising in the theory of the transformation of hyperelliptic functions has been studied by Klein, Burkhardt, Maschke, Witting, and others. Jordan $\dagger$ had already determined the Galois group of the equation for the 27 lines on a general cubic surface and proved\$ it isomorphic with the abelian group for the trisection of hyperelliptic functions of four periods. The possibility of the solution by hyperelliptic functions of the equation for the 27 lines on a cubic surface having been thus established, Klein§ proceeded to sketch a method of reduction of the problem of the equation of the 27 straight lines to the problem of the trisection of the hyperelliptic function of 1st order. The actual calculations were effected by Burkhardt, /| who gives full references to the work of Witting 9 on a configuration in space connected with the $G_{51840}$ and to that of Maschke** on the complete form system of the group.

From the obscurity of a passage on p. 214 of a note by $G$. G. Morrice $+\dagger$ some readers have inferred that the simplicity of the group of 51840 linear substitutions had been established. The group probably meant was the group of order 4 and the word "simplicity" is not used in its technical group sense.

Various linear congruence groups will be found in Part II which are isomorphic with the present $G_{51840}$. The latter group is a subgroup of index 28 under the group of order 1,451,520, the group of the equation of the 28 th de-

* Math. Annalen, vol. 52 (1899), pp. 243-270 ; Gött. Nach. 1897.

†Traité des substitutions, pp. 316-329 ; also Kühnen's Dissertation, Marburg, 1888.

$\ddagger$ Ibid, pp. 365-369; a shorter proof has been given by the writer in Comptes rendus, vol. 128 (1899), pp. 873-5.

\& Liouville's Journal (4), vol. 4 (1888), pp. 169-176.

|| Math. Annalen, vol. 38, and vol. 41, p. 312.

ๆ Gött. Diss., 1887 ; Math. Annalen, vol. 29.

** Math. Annalen, vol. 33, pp. 317-344.

†† Proc. Lond. Math. Soc., vol. 22 (1892), pp. 213-217. 
gree on which depends the theory of the double tangents to a curve of the fourth order.*

The determination of all surfaces of the third order which admit of collineations has been made by Karl Bobek. $\dagger$ The only surfaces found were those studied by Eckhardt. Among these is Clebsch's diagonal surface $\$$ with the group $G_{120}$; also surfaces with a $G_{648}$ and a $G_{108}$.

In the paper of Biermann,\| "Ueber die regelmässigen Punktgruppen in Räumen höherer Dimension und die zugehörigen linearen Substitutionen mehrerer Variabeln,", Klein's method for ordinary space is extended to $n$ dimensions and worked out for the case $n=5$. Schlegel had already determined the regular bodies in $R_{5}$. Biermann reached linear substitutions in two complex variables previously studied by Jordan and Klein.

Two special classes of finite linear groups of orders $n^{8}$ and $n^{4}\left(n^{2}-1\right)$ are given by Jordan. 9

\section{Part II.-Linear Groups in a Galois Field. \\ §3. Theory of the Galois Field.}

In a great many investigations in group theory, including the classic ones of Galois, Betti, Mathieu, Jordan, as well as in numerous more recent articles, the theory of Galois's imaginaries has proved to be an instrument of great power. Galois first studied the properties of the polynomials built on a root of a congruence of degree $n$ belonging to and irreducible modulo $p$. These $p^{n}$ distinct polynomials combine according to the ordinary laws of algebra. Let for example $j$ denote an (imaginary) root of the irreducible congruence

$$
x^{3}+x+1 \equiv 0(\bmod 2) \text {. }
$$

Then all rational functions of $j$ can be reduced to one of the eight forms

$$
0, \quad 1, j, j+1, \quad j^{2}, \quad j^{2}+1, \quad j^{2}+j, j^{2}+j+1 .
$$

The sum, difference, product, or quotient (except by zero) of any two of these polynomials reduced to an unique polynomial belonging to the set.

* Geiser, Math. Annalen, vol. 1, pp. 129-138.

Jordan, Traité des substitutions, p. 330; Comptes rendus, vol. 67 (1868).

Weber, Math. Annalen, vol. 23, pp. 489-503.

†Monatshefte für Math. und Physik. (Wien), vol. 10 (1899), pp. 102-122.

¥ Math. Annalen, vol. 10, p. 227.

\& Math. Annalen, vol. 4, p. 336.

|| Wien Ber., vol. 95, pp. 523-548.

đBull. S. $M$ F., vol. 5, pp. 175-177. 
Moore's abstract formulation of the theory is convenient for group investigations. In place of the above set of eight polynomials, Moore considers eight abstract marks constituting the Galois field of order 8 , in notation the $G F\left[2^{3}\right]$.

Rather elaborate expositions of the theory have been given by Schönemann, Dedekind, Serret, and Moore. A very complete, but elementary, exposition of the abstract theory of Galois fields, with proof of their existence, will be published shortly by the writer. Owing to the lack of references in the papers on the subject, the following bibliography on Galois fields has been added:

GaloIs: "Sur la théorie des nombres," Bulletin des sciences mathématiques, de M. Férussac, 1830 ; reprinted in Journal de mathématiques, 1846, and in Oeuvres mathématiques d'Evariste Galois, Paris, 1897.

SchönEmanN : "Grundzüge einer allgemeinen Theorie der höheren Congruenzen deren Modul eine reelle Primzahl ist," Crelle, vol. 31 (1846), pp. 269-325.

Dedekrnd: "Abriss einer Theorie der höheren Congruenzen in Bezug auf einen reellen Primzahl-Modulus." Crelle, vol. 54 (1857), pp. 1-26.

SERRET: "Sur les fonctions entières irréductibles suivant un module premier, dan le cas où le degré est une puissance du module," Journal de Liouville, 1873, p. 301 and p. 437; also in Cours d'algèbre supérieure, vol. 2, pp. 122-211.

Jordan: "Théorie de Galois," Traité des substitutions, 1870, pp. 14-18.

Pellet: "Sur les fonctions irréductibles suivant un module premier et une fonction modulaire," Comptes rendus, vol. 70 (1870), pp. 328-330, and vol. 93 (1881), p. 1065, also vol. 90 (1880), p. 1339.

Pellet: "Sur les fonctions réduites suivant un module premier," Bulletin Soc. Math. de France, vol. 17 (1889), pp. 156-167.

Pellet: "Sur la décomposition d'une fonction entière en facteurs irréductibles suivant un module premier $p$," Comptes rendus, vol. 86 (1878), p. 1071.

Mooke: "A doubly-infinite system of simple groups," Congress Mathematical Papers, 1893.

Borel et Drach : Théorie des nombres et algèbre supérieure, 1895.

Dickson : "Higher irreducible congruences," Bulletin, July, 1897, pp. 381-389. 


\section{\$4. The General Linear Homogeneous Group.}

Consider the $p^{n m}$ letters $l_{\xi_{1}}, \xi_{2}, \cdots, \xi_{m}$ characterized by $m$ indices, each being an arbitrary one of the $p^{n}$ marks of $G F\left[p^{n}\right]$. A linear homogeneous substitution (A) on these letters replaces $l_{\xi_{1}}, \cdots, \xi_{m}$ by $l_{\xi_{1}^{\prime}}, \cdots, \xi_{m^{\prime}}$ where

$$
\xi_{i}^{\prime}=\sum_{j=1}^{m} \alpha_{i j} \xi_{j} \quad(i=1, \cdots, m),
$$

the $\alpha_{i j}$ being certain marks of the $G F\left[p^{n}\right]$, such that the determinant $\left|\alpha_{i j}\right| \neq 0$.

Since the compound of two linear homogeneous substitutions is again a linear homogeneous substitution, we say that the totality of substitutions (A) form a group, the general linear homogeneous group of degree $p^{n m}$. In the linear form, this group is due to Jordan (Traite des substitutions, $\S 119, \S 169$ ), special forms being due to Galois. In the writer's dissertation* it was proven to be identical with a group of substitutions in one variable investigated by $\mathbf{E}$. Betti in 1852-1855 and in more detail by $\mathrm{E}$. Mathieu in 1861. Its structure (factors of composition) was determined by Jordan for the case $n=1$, and for general $n$ by the writer in his dissertation and somewhat later by Burnside. $\dagger$

\section{§5. The Linear Fractional Group.}

It is obtained analytically by taking the linear homogeneous group fractionally, so that its substitutions may be exhibited in the form

$$
\xi_{1}^{\prime}: \xi_{2}^{\prime}: \cdots: \xi_{m}^{\prime}=\sum_{j=1}^{m} \alpha_{1 j} \xi_{j}: \sum_{j=1}^{m} \alpha_{2 j} \xi_{j}: \cdots: \sum_{j=1}^{m} \alpha_{m j} \xi_{j} \text {. }
$$

It is a concrete form of the quotient group of the general linear homogenous group by the invariant subgroup formed of the substitutions.

$$
\xi_{i}^{\prime}=\mu \xi_{i}, \quad(i=1, \cdots, m) .
$$

For the case $m=2$, we have the ordinary linear fractional group in one variable. It was investigated by Galois, Mathieu, $\$$ etc.; its structure was determined for the case $n=1$ by Gierster\| and for general $n$ by Professor Moore

* Annals of Math., 1897, pp. 65-120, pp. 161-183.

+ The theory of groups, pp. 340-341.

$\ddagger$ Liouville's Journal (2) vol. 5, pp. 9-42 ; vol. 6, pp. 241-323.

II Math. Annalen, vol. 18 (1881), pp. 319-365.

8 The Congress Math. Papers of 1893 ; abstract in the Bulletin, Dec. 1893. 
and later, but imperfectly, by Professor Burnside.* The subgroups of the linear fractional group in the $G F\left[\mathrm{p}^{n}\right]$ were determined by Gierster for $n=1$ (Mathematische Annalen, vol. 18 (1881), pp. 319-365) and for general $n$ by Moore (Abstract in the BuLletin, Oct., 1898). It was shown that the subgroups of the modular group are eitker solvable groups or certain modular groups or finally groups of double the order of certain modular groups. The decomposition of the subgroups of the modular groups thus leads only to cyclic groups and modular groups.

The linear homogeneous group was proven by Moore (Bulletin, Nov., 1895) to be the group of holoedric transformation into itself of the regular abelian group of degree $p^{n}$. In the same paper were determined certain abstract defining invariants of the linear homogeneous and the linear fractional groups.

\section{\$6. The Abelian Linear Group.}

Consider two sets of $2 m$ indices

$$
\xi_{i 1}, \quad \eta_{i 1} ; \xi_{i 2}, \eta_{i 2} \quad(i=1,2, \cdots, m) .
$$

Of the substitutions of the general linear homogeneous group on $2 m$ indices in the $G F\left[p^{n}\right]$, those which, if operating simultaneously on the two sets of indices, multiply the function

$$
\zeta \equiv \sum_{i=1}^{m}\left|\begin{array}{ll}
\xi_{i 1} & \xi_{i 2} \\
\eta_{i 1} & \eta_{i 2}
\end{array}\right|
$$

by a constant factor, form a group, the general abelian group. We exclude the case $m=1$, when the group is merely the general binary linear homogeneous group treated in $\$ 4$.

Its structure was determined by Jordan $\dagger$ for the case $n=1$ and by the writer $\$$ for general $n$. Indeed, its factors of composition are the prime factors of $p^{n}-1$ together with the factors of composition of the special abelian group of those substitutions leaving $\zeta$ absolntely invariant. For $p=2$, the latter group is simple except when $m=2, n=1$, when it is isomorphic with the symmetric group on six letters. For $p>2$, it has a maximal self-conjugate subgroup of order 2 , so that the quotient group is simple.

$\S 7$. An immediate generalization of the abelian group consists in the group of substitutions on $m q$ variables

\footnotetext{
* Proc. Lond. Math. Soc., vol. 25 (Feb., 1894), pp. 113-139,.

† Traité des substitutions, pp. 171-179.

$\ddagger$ Quar. Jour. of Math., July, 1897, pp. 169-178, and April, 1899, pp. 383-4.
} 
PROGRESS IN THEORY OF LINEAR GROUPS. [Oct.,

$$
x_{i j}{ }^{\prime}=\sum_{k=1}^{m}\left(\alpha_{k 1}^{i j} x_{k 1}+\cdots+\alpha_{k q}^{i j} x_{k q}\right) \quad\left(\begin{array}{c}
i=1, \cdots, m \\
j=1, \cdots, q
\end{array}\right)
$$

which, if operating cogrediently upon $q$ independent sets of $m q$ variables, the $j$ th set of which is exhibited thus

$$
x_{i 1}^{(j)}, x_{i 2}^{(j)}, \cdots, \quad x_{i q}^{(j)} \quad(i=1 \cdots m)
$$

will leave invariant up to a constant factor the function

$$
\varphi=\sum_{i=1}^{m}\left|\begin{array}{llll}
x_{i 1}^{(1)} & x_{i 2}^{(1)} & \cdots & x_{i q}^{(1)} \\
x_{i 1}^{(2)} & x_{i 2}^{(2)} & \cdots & x_{i q}^{(2)} \\
\cdots \cdots & \cdots & \cdots \\
x_{i 1}^{(q)} & x_{i 2}^{(q)} & x_{i q}^{(q)}
\end{array}\right| .
$$

This group was indicated by Kronecker. $\dagger$ The continuous group of linear substitutions leaving $\varphi$ invariant was investigated by the writer in the Bullesin for May, 1897. The structure of the discontinuous group of substitutions in the $G F\left[p^{n}\right]$ which leave $\varphi$ absolutely invariant has been determined by the writer.* For $q>2$, a substitution leaving $\varphi$ invariant must leave each determinant in $\varphi$ separately invariant or at most permute these determinants.

\section{\$8. The First and Second Hypoabelian Groups.}

These groups are certain subgroups of the abelian linear group in the $G F\left[2^{n}\right]$. For $n=1$, they were defined and investigated at length by Jordan making use of his theory of "exposants d'échange."

By a suitable generalization of Jordan (Traité, p. 440, \$ 578), we can give the following elementary definition of the generalized hypoabelian groups. The first and second hypoabelian groups in the $G F\left[2^{n}\right]$ are the groups of linear homogeneous substitutions

$$
\begin{aligned}
& \xi_{i}^{\prime}=\sum_{j=1}^{m}\left(\alpha_{i} \xi_{j}+\gamma_{i j} \eta_{j}\right) \\
& \eta_{i}^{\prime}=\sum_{j=1}^{m}\left(\beta_{i j} \xi_{j}+\delta_{i} \eta_{j}\right)
\end{aligned} \quad(i=1, \cdots, m)
$$

which leave invariant the respective functions

$$
\sum_{i=1}^{m} \xi_{i} \eta_{i}, \sum_{i=1}^{m} \xi_{i} \eta_{i}+\lambda\left(\xi_{1}^{2}+\eta_{1}^{2}\right),
$$

where $\lambda$ is a mark such that $\xi_{1} \eta_{1}+\lambda\left(\xi_{1}{ }^{2}+\eta_{1}{ }^{2}\right)$ is an irreducible quadratic form in the field. The orders of the groups are respectively

†Compare Jordan, Traité des substitutions, p. 219.

* "A class of linear groups including the abelian group," Quar. Jour. of Math., July, 1899. 


$$
\begin{gathered}
2\left(2^{n m} \mp 1\right)\left[\left(2^{2 n(m-1)}-1\right) 2^{2 n(m-1)}\right] \\
{\left[\left(2^{2 n(m-2)}-1\right) 2^{2 n(m-2)}\right] \cdots\left[\left(2^{2 n}-1\right) 2^{2 n}\right] .}
\end{gathered}
$$

Each group has a subgroup of index two, defined by the invariant equation

$$
\sum_{i, j}^{1 \cdots} \alpha_{i j} \delta_{i j}+\lambda^{2}\left(\alpha_{11}{ }^{2}+\beta_{11}{ }^{2}+\gamma_{11}{ }^{2}+\delta_{11}{ }^{2}\right)=m,
$$

the terms in $\lambda^{2}$ not occurring for the first hypoabelian group. The subgroup is simple in the case of the first hypoabelian group if $m>2$, and for the second hypoabelian group if $m>1$. For $m=2$, * the former has two factors of composition each equal to $\left(2^{2 n}-1\right) 2^{n}$.

A definition of the abelian and the two hypoabelian groups as groups of isomorphisms of certain elementary groups has been given by the writer. $\dagger$ Consider, for example, the abstract group $F$ generated by the operators $\boldsymbol{\theta}, A_{1}, \cdots, A_{m}, B_{1}, \cdots, B_{m}$, all of period $p$ and mutually commutative except the pairs $A_{i}, B_{i}$ for which

$$
A_{i} B_{i}=\otimes B_{i} A_{i} \quad(i=1,2, \cdots, m) .
$$

The group of isomorphisms of $F$ is isomorphic with the abelian linear group for $p>2$ and with the first hypoabelian group for $p=2$.

\section{\$9. The Orthogonal Group.}

This group is formed of the linear homogeneous substitutions on $m$ indices $\xi_{1}, \ldots, \xi_{m}$ in the $G F\left[p^{n}\right], p>2$, which leave invariant the function $\sum_{i=1}^{m} \xi_{i}{ }^{2}$. Its order and generators were determined by Jordan $\ddagger$ for the case $n=1$, certain exceptional cases being overlooked, and by the writer§ for general $n$. It is generated by the folluwing substitutions [only those indices which are altered are written]:

$$
\begin{gathered}
C_{i}: \xi_{i}^{\prime}=-\xi_{i}, \\
O_{i j}^{\alpha \beta}:\left\{\begin{array}{l}
\xi_{i}^{\prime}=\alpha \xi_{i}+\beta \xi_{j}, \\
\xi_{j}^{\prime}=-\beta \xi_{i}+\alpha \xi_{j}, \quad\left(\alpha^{2}+\beta^{2}=1\right),
\end{array}\right.
\end{gathered}
$$

with two exceptional cases, when additional generators are necessary, viz.,

* Amer. Jour. of Math., July, 1899, p. 249.

$\dagger$ Abstract in the BuLletin, A pril, 1899; complete in the Transactions.

$\ddagger$ Traité des substitutions, pp. 155-177.

\& Bulletin, Feb., 1898. 


$$
\begin{aligned}
& \text { for } p^{n}=5, m \equiv 3, \quad R:\left\{\begin{array}{l}
\xi_{1}^{\prime}=\xi_{1}+\xi_{2}+2 \xi_{3} \\
\xi_{2}^{\prime}=\xi_{1}+2 \xi_{2}+\xi_{3} \\
\xi_{3}^{\prime}=2 \xi_{1}+\xi_{2}+\xi_{3}
\end{array}\right\} \quad\left(R^{2}=1\right), \\
& \text { for } p^{n}=3, m \equiv 4, \quad W:\left\{\begin{array}{l}
\xi_{1}^{\prime}=\xi_{1}-\xi_{2}-\xi_{3}-\xi_{4} \\
\xi_{2}^{\prime}=\xi_{1}-\xi_{2}+\xi_{3}+\xi_{4} \\
\xi_{3}^{\prime}=\xi_{1}+\xi_{2}-\xi_{3}+\xi_{4} \\
\xi_{4}^{\prime}=\xi_{1}+\xi_{2}+\xi_{3}-\xi_{4}
\end{array}\left(W^{3}=1\right) .\right.
\end{aligned}
$$

Its structure has been fully determined by the writer in several distinct manners. $*$ For $m$ odd and $>1$, the orthogonal group has the factors of composition 2,2 , and

$$
\frac{1}{2}\left(p^{n(m-1)}-1\right) p^{n(m-2)}\left(p^{n(m-3)}-1\right) p^{n(m-4)} \cdots\left(p^{2 n}-1\right) p^{n},
$$

the case $p^{n}=3, m=3$ being an exception, the factors being $2,2,2,2,3$.

For $m$ even and $>4$, it has the factors of composition $2,2,2$, and

$$
\begin{gathered}
\left.\frac{1}{4}\left[p^{n(m-1)}-\varepsilon^{\frac{m}{2}} p^{n}\left(\frac{m}{2}-1\right)\right]\left[\left(p^{n(m-2)}-1\right) p^{n(m-3)}\right)\right] \\
\cdots \cdot\left[\left(p^{2 n}-1\right) p^{n}\right],
\end{gathered}
$$

$\varepsilon_{\text {being }} \pm 1$ according to the form $4 l \pm 1$ of $p^{n}$.

For $m=4$, it has the factors of composition 2, 2, $\frac{1}{2}\left(p^{2 n}-1\right) p^{n}, \frac{1}{2}\left(p^{2 n}-1\right) p^{n}$ and 2 , except in the cases $p^{n}=2$ and 3 , when the composite numbers 6 and 12 respectively are to be replaced by their prime factors.

\section{\$10. Other Linear Groups with a Quadratic Invariant. $\dagger$}

Every $m$-ary linear group in the $G F\left[p^{n}\right], p>2$, defined by an invariant which is a quadratic form of determinant not zero in the field, can be transformed into the orthogonal group if $m$ be odd; while for $m$ even, it can be transformed either into the orthogonal group or else into the group leaving invariant the form

$$
\sum_{i=1}^{m-1} \xi_{i}{ }^{2}+\nu \xi_{m}^{2} \quad(\nu=\text { not-square }) .
$$

The latter group has for $m>2$, the factors of composition 2,2 , and

$$
\left.\frac{1}{2}\left[p^{n(m-1)}+\varepsilon^{\frac{m}{2}} p^{n}\left(\frac{m}{2}-1\right)\right]\left[\left(p^{n(m-2)}-1\right) p^{n(m-3)}\right)\right]
$$

* Proc. Calif. Acad. Sci. (3), vol. 1, nos. 4-5; Bulletra, May, 1898; Amer. Jour. of Math., July, 1899 ; Proc. Lond. Math. Soc., vol. 30, pp. 70-98.

†Amer. Jour. of Math., July, 1899. 


$$
\text { … }\left[\left(p^{2 n}-1\right) p^{n}\right] \text {. }
$$

For $m=2$, it is simply isomorphic with the linear fractional group in the $G F\left[p^{2 n}\right]$.

Every $m$-ary linear homogeneous group in the $G F\left[2^{n}\right]$ defined by a quadratic invariant, not expressible as a quadratic form in fewer than $m$ variables, can be transformed linearly into a group which, for $m$ even, is either the first or else the second hypoabelian group; while for $m$ odd, $m \equiv 2 M+1$, it is the group leaving invariant

$$
\xi_{0}^{2}+\sum_{i=1}^{M} \xi_{i} \eta_{i}
$$

The latter.group is simply isomorphic with the abelian group on $2 M$ indices in the $G F\left[2^{n}\right]$.

\section{\$11. Linear Group defined by the Invariant $\sum_{i=1}^{m} \xi_{i 1} \xi_{i 2} \cdots \xi_{i q^{*}}$}

For $q=2$, this group was studied at length by the writer (Proc. Lond. Math. Soc., vol. 30, pp. 70-98), and for $q>2$ (Ibid., pp. 200-208). For $q=2, p>2$, the group is isomorphic with the orthogonal group, except in the case $m$ odd and -1 a not-square in the field, when it is isomorphic with the group of $\S 10$. For $q>2$, the substit utions leave the products $\xi_{i 1} \xi_{i 2} \cdots \xi_{i q}(i=1, \cdots, m)$ separately invariant, or else merely permute them. Its structure is therefore evident.

\section{\$12. The Hyperorthogonal Group.}

In a paper contributed to the Mathematische Annalen, the writer has determined the order, generators, and structure of the linear homogeneous $m$-ary group defined by the invariant

$$
\varphi_{r} \equiv \sum_{r=1}^{m} \lambda_{i} \xi_{i}{ }^{r} \quad(r>2) \text {. }
$$

There exists no continuous group leaving invariant $\varphi r$ $(r>2)$, while the only collineations of $\varphi_{r}=0$ into itself are those leaving the terms $\lambda_{1} \xi_{1}{ }^{r}, \cdots, \lambda_{m} \xi_{m}{ }^{r}$ separately fixed, or at most permuting them. The latter result holds for linear substitutions in the $G F\left[p^{n}\right]$, if $r$ be neither a multiple of $p$ nor of the form $p^{s}+1$. If $r$ be of either of these two forms, the determination of the group reduces to that of the group in the $G F\left[p^{2 s}\right]$ leaving invariant $\sum_{i=1}^{m} \xi_{i}{ }^{s}+1$. The latter group may be called the Hyperorthogonal Group, since those of its substitutions which belong to the $G F\left[p^{s}\right]$ form the orthogonal group in the $G F\left[p^{s}\right]$. 

tions

The hyperorthogonal group is generated by the substitu-

$$
\begin{aligned}
& T_{i, \tau}: \quad \xi_{i}^{\prime}=\tau \xi_{i} \\
& \left(\tau^{p^{8}+1}=1\right), \\
& O_{i j}^{a \beta}:\left\{\begin{array}{l}
\xi_{i}^{\prime}=\alpha \xi_{i}+\beta \xi_{j} \\
\xi_{j}^{\prime}=-\beta^{p^{8}} \xi_{i}+\alpha^{p^{8}} \xi_{j}
\end{array}\right. \\
& \left(\alpha^{p^{s}+1}+\beta^{p^{s}+1}=1\right) \text {, }
\end{aligned}
$$

except for the case $p^{s}=2, m \equiv 3$, when a new generator is. necessary, for example,

$$
W:\left(\begin{array}{ccc}
1 & 1 & 1 \\
1 & i & i^{2} \\
1 & i^{2} & i
\end{array}\right) \quad\left[i^{2}+i+01 \equiv 0(\bmod 2)\right]
$$

Those of its substitutions having determinant unity form a self-conjugate subgroup of index $p^{s}+1$. The factors of composition of this subgroup are the prime factors of $d$ and the composite number

$$
\begin{gathered}
\frac{1}{d}\left[p^{s m}-(-1)^{m}\right] p^{s(m-1)}\left[p^{s(m-1)}-(-1)^{m-1}\right] \\
p^{s(m-2)} \cdots\left[p^{2 s}-1\right] p^{s},
\end{gathered}
$$

where $d$ is the greatest common divisor of $m$ and $p^{8}+1$. The cases $m=3, p^{s}=2 ; m=2, p^{s}=2 ; m=2, p^{s}=3$ are exceptional. For $m=2$, the linear substitutions in the $G F$ $\left[p^{2 s}\right]$ which have determined unity and which leave invariant $\xi_{1}^{p^{s}+1}+\xi_{2}^{p s}+1$ form a group isomorphic with the linear fractional group in the $G F\left[p^{s}\right]$.

\section{\$13. The Hyperabelian group.}

This group consists of the totality of linear homogeneous substitutions on $2 m$ indices in the $G F\left[p^{2 n}\right]$ which leave invariant

$$
\psi \equiv \sum_{i=1}^{m}\left(\xi_{2 i-1} \xi_{2 i}^{p^{n}}-\xi_{2 i} \xi_{2 i-1}^{p^{n}}\right) .
$$

The totality of substitutions with coefficients in the $G F\left[p^{n}\right]$ which leave $\psi$ invariant forms a subgroup which is readily recognized as the abelian linear group on $2 m$ indices in the $G F\left[p^{n}\right]$. Hence the name hyperabelian.* It is easily proven to be simply isomorphic with the hyperorthogonal group on $2 m$ indices in the $G F\left[p^{2 n}\right]$.

* Dickson: "Concerning the four known simple groups of order 25920, with an introduction to the hyperabelian group," Proc. Lond. Math. Soc., vol. 30 (1899). 


\section{\$14. Isomorphisms between Various Iinear Groups.}

The conception of "the compounds of a given linear homogeneous group," introduced in recent papers by the writer, * has proven to be a powerful means of setting up isomorphisms. The group of quaternary linear homogeneous substitutions of determinant unity in the $G F\left[p^{n}\right]$ is isomorphic with a subgroup of the senary linear group leaving invariant $\xi_{1} \eta_{1}+\xi_{2} \eta_{2}+\xi_{3} \eta_{3}$, and hence, according as $p^{n}=2^{n}$, $4 l+1$, or $4 l-1$, to the senary first hypoabelian group, the senary orthogonal group, or the group of $\S 10$.

The simple group of order $\frac{1}{2}\left(p^{4 n}-1\right)\left(p^{2 n}-1\right) p^{4 n}$ derived from the quaternary abelian group and that from the quinary orthogonal group, each in the $G F\left[p^{n}\right], p>2$, are simply isomorphic.

The following four simple groups of order 25920 are simply isomorphic: $\dagger$

$1^{\circ}$. The abelian group on four indices modulo 3 .

$2^{\circ}$. The second hypoabelian group on six indices modulo 2 .

$3^{\circ}$. The orthogonal group on five indices modulo 3 .

$4^{\circ}$. The hyperabelian group on four indices in the $G F\left[2^{2}\right]$.

In the paper cited an abstract group, a substitution-group on 36 letters, and one on 27 letters are given, each isomorphic to the above groups of order 25920 .

\section{SHORTER NOTICES.}

Leçons sur la détermination des orbites, professées à la Faculté des Sciences de Paris. Par F. Tisserand ; redigées et developpées pour les calculs numériques, par J. Ревснот ; avec une Préface de H. Porncaré. Paris, GauthierVillars et Fils. 1899. 4to, xiv + $124 \mathrm{pp}$.

Tisserand is chiefly known outside of France for his Traité de mécanique céleste-a monument of labor undertaken and carried out amid the pressure of many duties. He had in fact an enormous capacity for work and in his numerous memoirs left few parts of theoretical astronomy untouched. The volume of 120 quarto pages before us, edited by M. Perchot, shows him working for the practical

* "Concerning a linear homogeneous group in $C_{m, q}$ variables isomorphic to the general linear homogeneous group in $m$ variables," BULLETIN, Dec., 1898. "The structure of certain linear groups with quadratic invariants," Proc. Lond. Math. Soc., vol. 30, pp. 70-98. "A new definition of the general abelian linear group," Transactions (read Feb. 1899).

† Proc. Lond. Math. Soc., vol. 31. Abstract in Bulceris, May, 1899, p. 384 . 